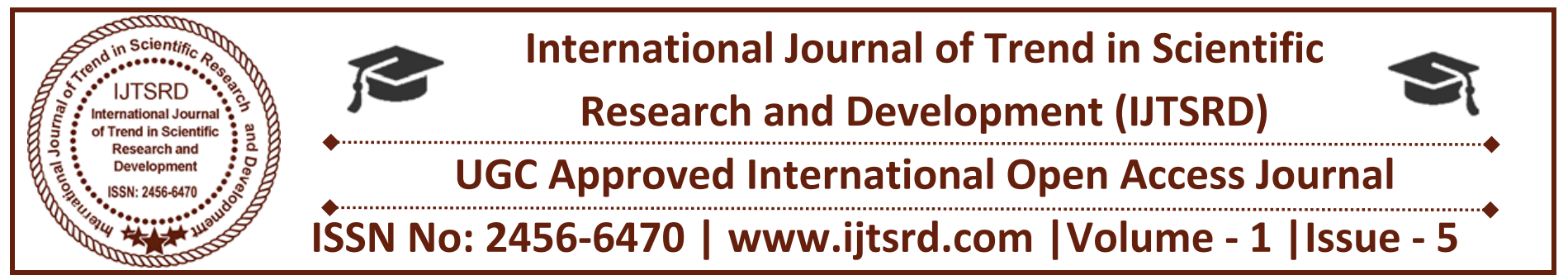

\title{
MIMO Systems with Degraded Actuators and Sensors
}

\author{
Chieh-Chuan Feng \\ Department of Electrical Engineering \\ I-Shou University \\ Kaohsiung City 84001, Taiwan
}

\begin{abstract}
This paper presents a reliable control design technique for linear, time-invariant, multi-input multi-output (MIMO) systems with degraded actuators and sensors. The degradation defined in this paper ranges from normal operational conditions to complete failure of actuators and sensors. We derive linear matrix inequality (LMI) conditions ensuring robust stability of the system using static state feedback. The potential of the proposed technique has been demonstrated by an example of three coupled inverted pendulums.
\end{abstract}

\section{Keywords-linear matrix inequality; static state feedback; MIMO; degradation.}

\section{Introduction}

Conventional feedback control designs for a MIMO system may result in instability in the event of the degradation of actuators and sensors, even though it may be possible to control the system using only the surviving functions of actuator and sensor. It is worth noting that this condition can occur even if the open loop system is stable. It is therefore of interest to develop feedback control designs which guarantee robust stability despite degradation of actuators and sensors.

There are relatively few methods for design of reliable control. Veillette et al [1] develop observer-based reliable centralized and decentralized control systems provided the failure of actuators and sensors occur only within a prespecified subset of the control components. In addition, the actuators and sensors are subject to either failure or normal operation which neglects the possibility of partial degradation. Fujita and Shimemura [2] provide necessary and sufficient conditions by introducing a class of $\boldsymbol{U}$-matrices for examining system stability against arbitrary feedbackloop failures in multivariable control systems with a stable plant and controller. This condition requires an exponential growth in computation as a function of plant dimension. Partial failures are not considered. For a given stabilizing controller of a plant, Vidyasagar and Viswanadham [3] studied the problem of designing the second controller such that either controller acting alone stabilizes the plant and both acting together also stabilize the plant. A computing method for the second stabilizing controller which involves stable coprime factorizations using the plant and the first controller data are established and may result in the second controller of high order. Cho et al [4] used the same methodology as [3] except that the second stabilizing controller is of adaptive controller which again ends up with high order.

In this paper, we design a static state feedback control system to tolerate the degradation ranging from normal operational condition to complete failure of actuator and sensor. The degradation is modeled as a multiplicative uncertainty at the plant input or output. We accomplish this via diagonal weighting and norm 
bounded matrices. To simplify the derivation we will first develop and prove LMI conditions which tolerate sensor or actuator degradation independently. Our approach is extended to the simultaneous degradation actuators and sensors in the system.

\section{Problem Formulation}

The purpose of this section is to define the framework on which our approach of reliable control systems is based. The degradation for actuators and sensors will be considered separately to simplify the derivation.

\section{A. Actuator case:}

Actuator case represents the degradation of actuators and is shown as follows,

$$
\begin{aligned}
& \dot{x}=A x+B u \\
& u=u_{k}+p_{u} \\
& q_{u}=\varphi_{u} u_{k} \\
& p_{u}^{r}=\delta_{u}^{r} q_{u}^{r}, \quad\left|\delta_{u}^{r}(t)\right| \leq 1 .
\end{aligned}
$$

where $x \in R^{n}$ denotes the state of the system, $u_{k} \in R^{m}$ is the output of the control gain shown in Figure 1. $u \in$ $R^{m}$ is the input to the plant. $\varphi_{u}$ is the uncertainty weighting of the control input to the plant. Note that $p_{u}$ and $q_{u}$ represent the output and input vectors of plant input perturbations. $p_{u}{ }^{r}$ and $q_{u}{ }^{r}$ correspond to the $r$ th element of vector $p_{u}$ and $q_{u}$ respectively.

$$
\begin{array}{rlrl}
p_{u} & =\left[\begin{array}{lllll}
p_{u}{ }^{1} \ldots & \ldots & p_{u}{ }^{r} \ldots & p_{u}{ }^{m}
\end{array}\right]^{T}, & & p_{u}^{r} \in R \\
q_{u} & =\left[\begin{array}{llllll}
q_{u}{ }^{1} \ldots & q_{u}{ }^{r} \ldots & q_{u}{ }^{m}
\end{array}\right]^{T}, & q_{u}{ }^{r} \in R \\
\varphi_{u} & =\operatorname{diag}\left(\varphi_{u}{ }^{1} \ldots . \varphi_{u}{ }^{r} \ldots \varphi_{u}{ }^{m}\right), & & \varphi_{u}{ }^{r} \in R
\end{array}
$$

$\delta_{u}^{r}(t)$ is the real valued unknown bounded uncertainty and it is assumed to be time-varying scalar quantity. The control signal $u(t)$ can be readily computed as

$$
u(t)=\left(I+\delta_{u}(t) \varphi_{u}\right) u_{k}(t)
$$

where the $I$ is identity matrix and $\delta_{u}=\operatorname{diag}\left(\delta_{u}{ }^{1} \ldots . \delta_{u}{ }^{r} \ldots\right.$ $\left.\delta_{u}{ }^{m}\right), \quad \delta_{u}^{r} \in R$. Since $\left|\delta_{u}^{r}(\mathrm{t})\right| \leq 1$, i.e. $-1 \leq \delta_{u}^{r}(t) \leq 1$, the degradation is modeled by a priori weighting $\varphi_{u}$ as follows.

(1) $\varphi_{u}^{r}=1$ indicates the $r$ th actuator may fail fully.

(2) $\varphi_{u}^{r}=0$ indicates there is no possibility that the $r$ th actuator fails partially or fully.
(3) $0<\varphi_{u}^{r}<1$ indicates the $r$ th actuator may fail partially.

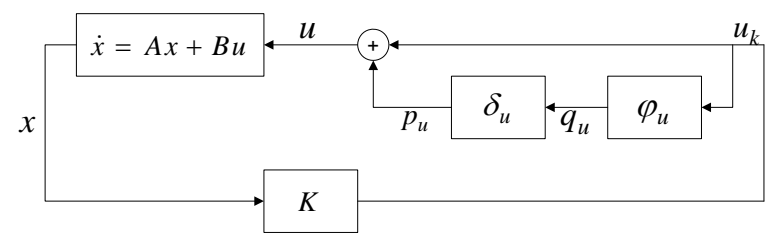

Figure 1 Closed Loop System for Actuator Case

\section{B. Sensor case:}

Sensor case represents the degradation of sensors and is written as follows,

$$
\begin{aligned}
& \dot{x}=A x+B u \\
& x_{s}=x+p_{s} \\
& q_{s}=\psi_{s} x \\
& p_{s}^{l}=\delta_{s}^{l} q_{s}^{l}, \quad\left|\delta_{s}^{l}(t)\right| \leq 1 .
\end{aligned}
$$

where $\psi_{s}$ is the uncertainty weighting of the sensor and $x_{s}$ denotes sensor output. The definition for $p_{s}, q_{s}, \psi_{s}$, and $\delta_{\text {s }}$ are defined in (2.3) in a similar way as in the actuator case and can be seen clearly from Figure 2 where it shows the closed-loop system for sensor degradation.

$$
\begin{aligned}
& p_{s}=\left[p_{s}{ }^{1} \ldots p_{s}{ }^{l} \ldots p_{s}{ }^{n}\right]^{T}, \quad p_{s}{ }^{l} \in R \\
& q_{s}=\left[\begin{array}{lllll}
q_{s}{ }^{1} & \ldots & q_{s}{ }^{l} \ldots & q_{s}{ }^{n}
\end{array}\right]^{T}, \quad q_{s}{ }^{l} \in R \\
& \delta_{s}=\operatorname{diag}\left(\delta_{s}{ }^{1} \ldots \delta_{s}^{l} \ldots \delta_{s}^{n}\right), \quad \delta_{s}^{l} \in R \\
& \psi_{s}=\operatorname{diag}\left(\psi_{s}{ }^{1} \ldots \psi_{s}{ }^{l} \ldots \psi_{s}{ }^{n}\right), \quad \psi_{s}^{l} \in R
\end{aligned}
$$

The degraded sensor signal $x_{s}(t)$ can be readily represented as

$$
X_{s}(t)=\left(I+\delta_{s}(t) \psi_{s}\right) x(t)
$$

where the possibility of full or partial failure of sensors is defined in the similar way as in the actuator case using variables $\delta_{s}$ and $\psi_{s}$ in place of $\delta_{u}$ and $\varphi_{u}$, respectively.

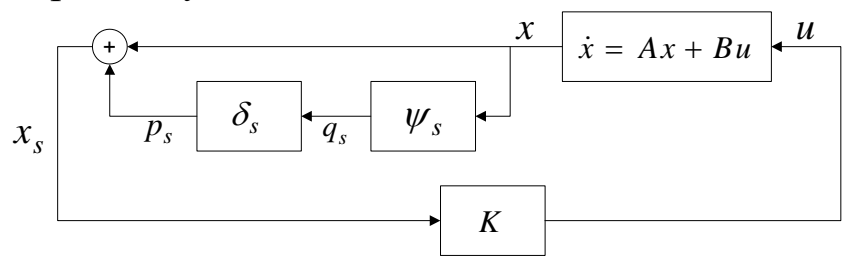

Figure 2 Closed Loop System for Sensor Case 
International Journal of Trend in Scientific Research and Development (IJTSRD) ISSN: 2456-6470

\section{Actuator and sensor case}

Actuator and sensor case is the case where actuator and sensor are simultaneously degraded shown as follows,

$$
\begin{array}{ll}
\dot{x}=A x+B u \\
u=u_{k}+p_{u} \\
x_{s}=x+p_{s} \\
q_{u}=\varphi_{u} u_{k} \\
q_{s}=\psi_{s} x \\
p_{u}^{r}=\delta_{u}^{r} q_{u}^{r}, & \left|\delta_{u}^{r}(t)\right| \leq 1 \\
p_{s}^{l}=\delta_{s}^{l} q_{s}^{l}, & \left|\delta_{s}^{l}(t)\right| \leq 1 .
\end{array}
$$

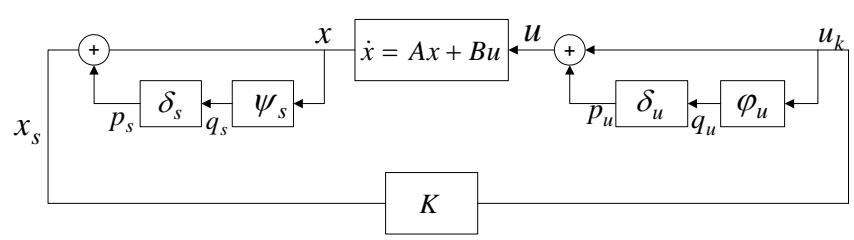

Figure 3 Closed-loop System for

Actuator and Sensor Case

The matrices $A$ and $B$ shown in (2.1), (2.2), and (2.3) are assumed to be real constant matrices with appropriate dimensions. $\varphi u$ and $\psi_{s}$ are given a priori and represent the uncertainty weighting used to model the degradation for actuators and sensors. We define

$$
\begin{aligned}
\Delta_{u}:=\operatorname{diag}\left(\delta_{u}^{r}(t)\right)_{r=1, \ldots, m,} & \delta_{u}^{r}(t) \in R \\
\Delta_{s}:=\operatorname{diag}\left(\delta_{s}^{l}(t)\right)_{l=1, \ldots, n,} & \delta_{s}^{l}(t) \in R
\end{aligned}
$$

where $\left\|\Delta_{u}\right\|_{\infty} \leq 1$ and $\left\|\Delta_{s}\right\|_{\infty} \leq 1$.

The control problem is to find, if it exists, static state feedback that stabilizes the system described in (2.1), (2.2), or (2.3).

\section{Controller Synthesis}

In this section we present the main results. Lyapunov stability theory is used to establish stability criteria. Although there is no trivial method to establish a Lyapunov function, by experience, quadratic Lyapunov functions, i.e. $V(\xi)=\xi^{T} L \xi$, have been proven to be efficient and easily implemented [7]. We consider static state feedback, i.e. $u_{k}(t)=K x(t)$ for the actuator case, $u(t)=K x_{s}(t)$ for sensor case and $u k(t)=$ $K x_{s}(t)$ for combination of actuator and sensor case.
Theorem 1. Consider the closed-loop system

$$
\begin{aligned}
& \dot{x}=(A+B K) x+B p_{u} \\
& q_{u}=\varphi_{u} K x \\
& p_{u}^{r}=\delta_{u}^{r} q_{u}^{r}, \quad\left|\delta_{u}^{r}(t)\right| \leq 1 .
\end{aligned}
$$

If there exist matrices $Q, Y$, and $M$ satisfying

$$
\begin{gathered}
Q=Q^{T}>0 \\
{\left[\begin{array}{cc}
Q A^{T}+A Q+B Y+Y^{T} B^{T}+B M B^{T} & Y^{T} \varphi_{u}^{T} \\
\varphi_{u} Y & -M
\end{array}\right]<0}
\end{gathered}
$$

for a given weighting matrix $\varphi_{u}$, then the following statements are equivalent,

(1) The closed-loop system (3.1) is asymptotically stable.

(2) $(A+B K)$ is Hurwitz for $K=Y Q^{-1}$.

Proof. See Appendix A for proof and notation.

Remark 1 . The inequality (3.2) is convex in the matrix variables $Q, Y$, and $M$. Thus, (3.2) can be readily solved by convex optimization techniques. We write a feasibility problem

Find

$Q, Y$, and $M$

Subject to $Q=Q^{\mathrm{T}}>0, M>0$, and (3.2).

Theorem 2. Consider the closed-loop system

$$
\begin{aligned}
& \dot{x}=(A+B K) x+B K p_{s} \\
& q_{s}=\psi_{s} x \\
& p_{s}^{l}=\delta_{s}^{l} q_{s}^{l}, \quad\left|\delta_{s}^{l}(t)\right| \leq 1 .
\end{aligned}
$$

If there exist matrices $P, W, S$ and $\Lambda$ satisfying

$$
\begin{gathered}
P=P^{T}>0, \quad S=S^{T}>0, \quad \Lambda>0 \\
-P \Lambda P+W^{T} S^{-1} W<0 \\
{\left[\begin{array}{cc}
P A^{T}+A P+W^{T} B^{T}+B W+B S B^{T} & P \psi_{s}^{T} \\
\psi_{s} P & -\Lambda^{-1}
\end{array}\right]<0}
\end{gathered}
$$

for a given weighting matrix $\psi_{s}$, then the following statements are equivalent.

(1) The closed-loop system (3.3) is asymptotically stable.

(2) $(A+B K)$ is Hurwitz for $K=W P^{-1}$.

Proof. See proof for Theorem 3. 
Remark 2. The matrix inequalities (3.4) and (3.5) are inversely coupled through the matrices $S$ and $\Lambda$. Moreover the inequality (3.4) is not an LMI. Thus, to solve theorem 2 simultaneously through the use of convex optimization is difficult. However, we may still solve the problem using of sequential method that we solve for (3.5) first and then verify (3.4).

Theorem 3. Consider the following closed-loop system

$$
\begin{aligned}
& \dot{x}=(A+B K) x+B K p_{s}+B p_{u} \\
& q_{u}=\varphi_{u} K\left(x+p_{s}\right) \\
& q_{s}=\psi_{s} x \\
& p_{u}^{r}=\delta_{u}^{r} q_{u}^{r}, \quad\left|\delta_{u}^{r}(t)\right| \leq 1 \\
& p_{s}^{l}=\delta_{s}^{l} q_{s}^{l}, \quad\left|\delta_{\mathrm{s}}^{l}(t)\right| \leq 1 .
\end{aligned}
$$

If there exist matrices $P, W, S_{u}, S_{x}, S_{s}, \Lambda$, and $\Gamma$ satisfying

$$
\begin{aligned}
& P=P^{T}>0, S_{u}=S_{u}^{T}>0, S_{x}=S_{x}^{T}>0, S_{s}=S_{s}^{T}>0 \\
& \Lambda>0, \Gamma>0 \\
& -\Lambda+B^{T} S_{u}^{-1} B<0 \\
& -P \Lambda P+W^{T}\left(S_{x}^{-1}+S_{s}^{-1}+\Phi_{\Gamma}\right) W<0 \\
& {\left[\begin{array}{ccc}
P A^{T}+A P+W^{T} B^{T}+B W \\
+B S_{x} B^{T}+S_{u}
\end{array}\right)} \\
& \left.\begin{array}{ccc}
W & W^{T} & P \psi_{s}^{T} \\
\psi_{s} P & -\left(\Phi_{\Gamma}^{T} S_{s} \Phi_{\Gamma}+\Phi_{\Gamma}\right)^{-1} & 0 \\
& 0 & -\Lambda^{-1}
\end{array}\right]<0
\end{aligned}
$$

where $\Phi_{\Gamma}=\varphi_{u}^{T} \Gamma \varphi_{u}$, for a given weighting matrix $\varphi_{u}$ and $\psi_{\mathrm{s}}$, then the following statements are equivalent,

(1) The closed-loop system (3.6) is asymptotically stable.

(2) $(A+B K)$ is Hurwitz for $K=W P^{-1}$.

Proof. See Appendix B for proof.

Remark 3. Normally speaking, the matrix inequalities (3.6)-(3.8) should be solved simultaneously. However, it is not possible to solve using convex programming due to inversely coupled terms, $S_{s}, S_{u}, S_{x}$, and $\Lambda$. Thus, we suggest for some given $\Phi_{\Gamma}$ we solve (3.8) for $P, W, S_{x}, S_{s}$, and $S_{u}$ and then verify (3.6) and (3.7).

\section{Numerical Example}

Consider the system in Figure 3 consisting of three coupled inverted pendulums of point masses $m_{i}$, and length $l_{i}$. The pendulums interact via three springs and three dampers of stiffness $k_{i j}$ and damping $b_{i j} ; i, j=$ $1,2,3$, and $i \neq j$. The distances from attached point of springs and dampers to the platform baseline are $a_{i}$. The system data are shown in Table 1. Using this system, we will demonstrate several examples for actuator and sensor degradation. The system dynamics for pendulums are written in the general form,

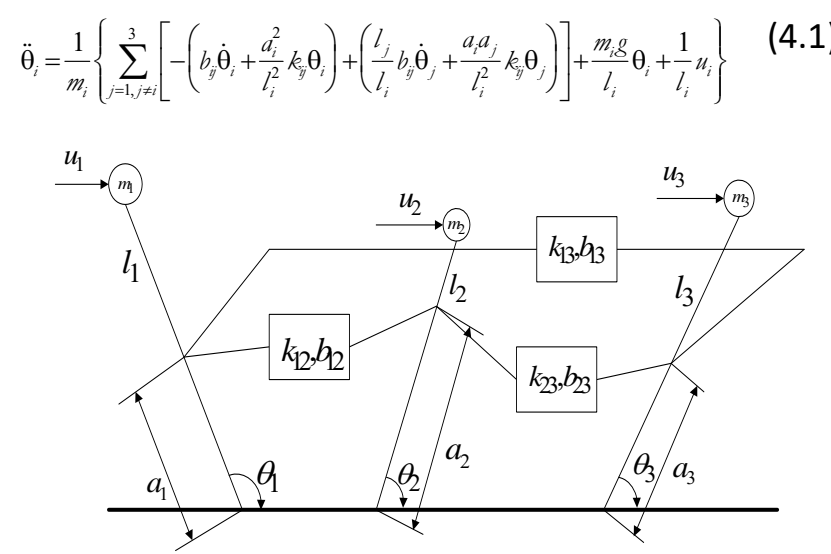

Figure 3 Three Inverted Pendulums Systems

Table 1

\begin{tabular}{|c|c|c|c|c|c|c|c|c|}
\hline$m_{1}$ & $m_{2}$ & $m_{3}$ & $b_{12}$ & $b_{13}$ & $b_{23}$ & $k_{12}$ & $k_{13}$ & $k_{23}$ \\
\hline 1 & 1 & 1 & 1 & .51 & 1 & 1 & .51 & 1 \\
\hline \multicolumn{10}{c|}{$a_{1}=a_{2}=a_{3}=0.5, \mathrm{~g}=10, l_{1}=1, l_{2}=1.2, l_{3}=1.1}$.
\end{tabular}

Initial conditions:

$\theta_{1}=-0.1, \dot{\theta}_{1}=0.11, \theta_{2}=0.34, \dot{\theta}_{2}=-0.23, \theta_{3}=-0.29, \dot{\theta}_{3}=-1.33$.

We will first demonstrate actuator degradation. The weighting is chosen $\varphi_{u}=\operatorname{diag}(1,0.1,0.28)$ which represents the possibility of degradation for each actuator. For instance, actuator for $m_{1}$ may fail during its operation, actuators for $m_{2}$ and $m_{3}$ are subject to $10 \%$ and $28 \%$ variation of its nominal operation signal. We will demonstrate nominal operation of actuators, i.e. $\varphi_{u}=0$, at time $t \leq 0.5$. Then, we will show the case where the actuator for $m_{1}$ is subject to fail and the actuator for $m_{2}$ and $m_{3}$ are partial failure. Detail conditions are shown in Table 2.

Table 2

\begin{tabular}{|c|c|c|c|}
\hline time & $m_{1}$ & $m_{2}$ & $m_{3}$ \\
\hline $0 \leq t<0.5$ & $\mathrm{~S}_{1}$ & $\mathrm{~S}_{2}$ & $\mathrm{~S}_{3}$ \\
\hline
\end{tabular}


International Journal of Trend in Scientific Research and Development (IJTSRD) ISSN: 2456-6470

\begin{tabular}{|c|c|c|c|}
\hline $0.5 \leq t<2.5$ & failure & $0.9 * S_{2}$ & $0.72 * S_{3}$ \\
\hline $2.5 \leq t \leq 6$ & $2 * S_{1}$ & $1.1 * S_{2}$ & $1.28 * S_{3}$ \\
\hline
\end{tabular}

chosen $\psi_{s}=\operatorname{diag}(0.4,0.5,0.4,1,0.5,1)$ which represents the possibility of

where $\mathrm{S}_{i}=$ the nominal operation control signal of actuator associated with each mass. The computed gain followed by Theorem 1 is

$K=\left[\begin{array}{cccccc}-44.6423 & -11.3417 & 2.7025 & -1.3565 & 1.2500 & -0.7005 \\ -362.5824 & -93.3584 & -2.9357 & -17.3282 & -4.3807 & -10.9399 \\ -25.4243 & -7.1539 & 4.5474 & -1.6523 & -35.8191 & -10.0032\end{array}\right]$

eigenvalues of $(A+B K)=\left[\begin{array}{ll}-45.2643 & -3.6514\end{array}\right.$

$1.1316 \pm 1.6097 \mathrm{i}-2.1306 \pm 2.1394 \mathrm{i}]$. The simulation results are shown in Figure 4 and 5 for plant outputs and control signals. It is noticed that the control signal for $m_{1}$ decays relatively fast at the beginning of operation and then is close to zero as shown in Figure 4. Thus, even though the actuator \#1 fails at time $t \geq$ $0.5 \mathrm{sec}$, it has very little effect to the system. Since the actuator signals for $m_{1}$ varies from twice of its nominal value to zero (which is used to represent the failure of the actuator), one possible way to accommodate such a large variation is simply to make the resultant signal as small as possible such that it has the least effect on the system. The jumps shown in Figure 5 are due to the switching at time $t=0.5 \mathrm{sec}$ instantaneously. If there are two actuators in the system subject to the possibilities of failure, e.g. $\varphi_{u}=\operatorname{diag}(1,1,0)$, we are not able to find feasible solutions for the Theorem 1. In other words, it is not possible to maintain stability of the system when two actuators failures in the system.

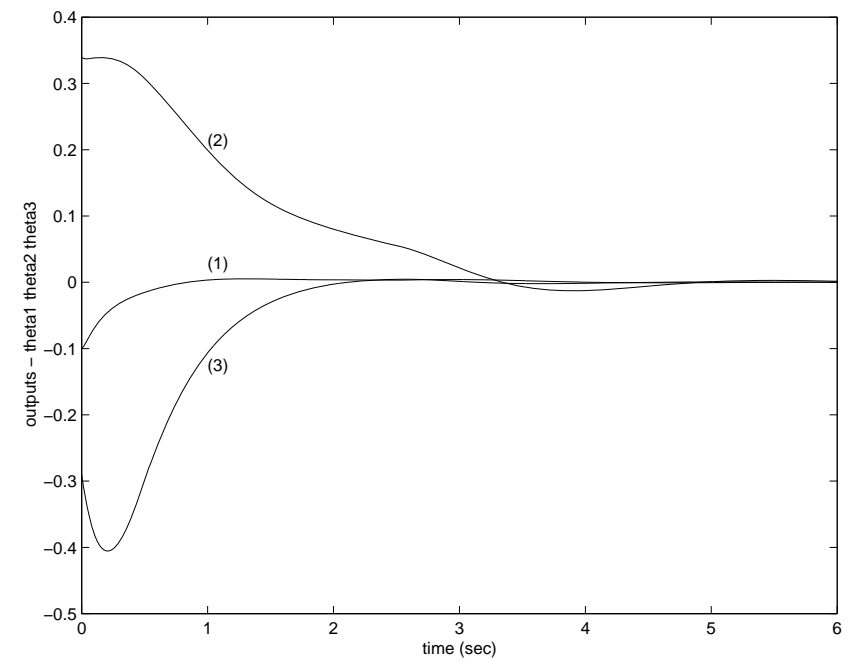

Figure 4 Outputs of Three Pendulums

For the sensor degradation case, the weighting is

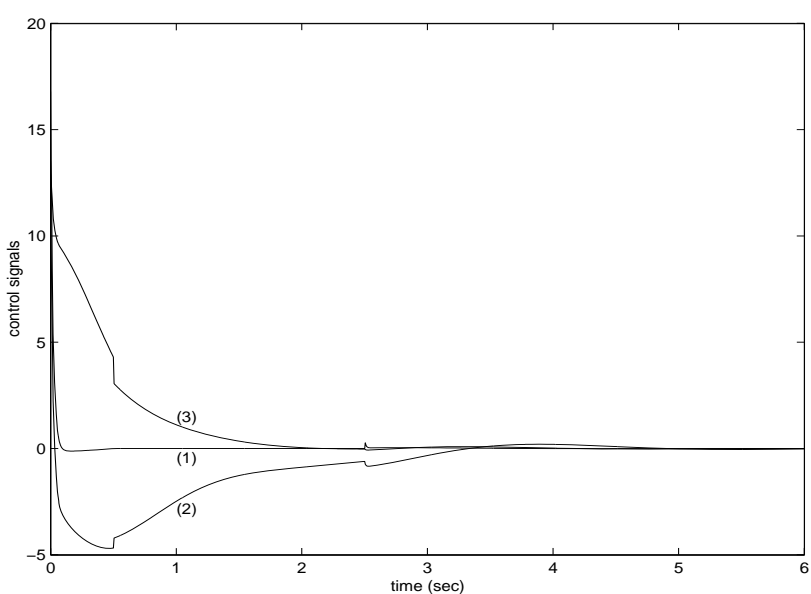

Figure 5 Control Signals

degradation for each sensor. The velocity sensor of $m_{1}$ is subject ++ to $50 \%$ variation, while the position sensor has $40 \%$ variation. The velocity sensors of $m_{2}$ and $m_{3}$ are subject to the possibility of failure, while the corresponding position sensors have $40 \%$ and $50 \%$ variations. We will demonstrate the extreme case, which is shown in Table 3. The computed gain followed by Theorem 2 is

$K=\left[\begin{array}{cccccc}-29.7241 & -8.2556 & 1.0801 & -1.0596 & 1.2811 & 0.3397 \\ 0.5669 & -0.9255 & -29.5401 & -8.0746 & 0.9343 & 0.9577 \\ 1.2812 & -0.2544 & 1.2151 & -0.9838 & -29.3428 & 8.3852\end{array}\right]$

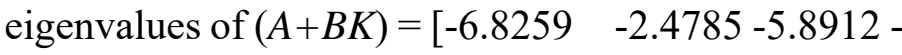
$3.0449-3.0952-6.2916]$. The simulation results are shown in Figure 6 and 7. The sudden jumps in Figure 7 show the instantaneous switching at time $t=0.5 \mathrm{sec}$. The following results are observed from simulation. The system will oscillate if we fail three velocity sensors. Since the damping control signals given by velocity sensor are zero, the occurrence of oscillation is not unexpected.

Table 3

\begin{tabular}{|c|c|c|c|c|c|c|}
\hline time & \multicolumn{2}{|c|}{$m_{1}$} & \multicolumn{2}{c|}{$m_{2}$} & \multicolumn{2}{c|}{$m_{3}$} \\
\hline & Pos & Vel & Pos & Vel & Pos & Vel \\
\hline $0 \leq t$ & $1.4^{*}$ & $1.5^{*}$ & $1.4^{*}$ & $2^{*}$ & $1.5^{*}$ & $2^{*}$ \\
$\leq .5$ & $\mathrm{~S}_{1 \mathrm{p}}$ & $\mathrm{S}_{1 \mathrm{v}}$ & $\mathrm{S}_{2 \mathrm{p}}$ & $\mathrm{S}_{2 \mathrm{v}}$ & $\mathrm{S}_{3 \mathrm{p}}$ & $\mathrm{S}_{3 \mathrm{v}}$ \\
\hline $0.5 \leq$ & $0.6^{*}$ & $0.5^{*}$ & $0.6^{*}$ & $\mathrm{~F}$ & $0.5^{*}$ & $\mathrm{~F}$ \\
$t \leq 5$ & $\mathrm{~S}_{1 \mathrm{p}}$ & $\mathrm{S}_{1 \mathrm{v}}$ & $\mathrm{S}_{2 \mathrm{p}}$ & & $\mathrm{S}_{3 \mathrm{p}}$ & \\
\hline
\end{tabular}

Pos $=$ position sensor, $\mathrm{Vel}=$ velocity sensor, $\mathrm{S}$ with all 
subscripts $=$ the nominal operational signal associated with each sensor (subscript $\mathrm{p}=$ position sensor, subscript $\mathrm{v}=$ velocity sensor). $\mathrm{F}=$ failure.

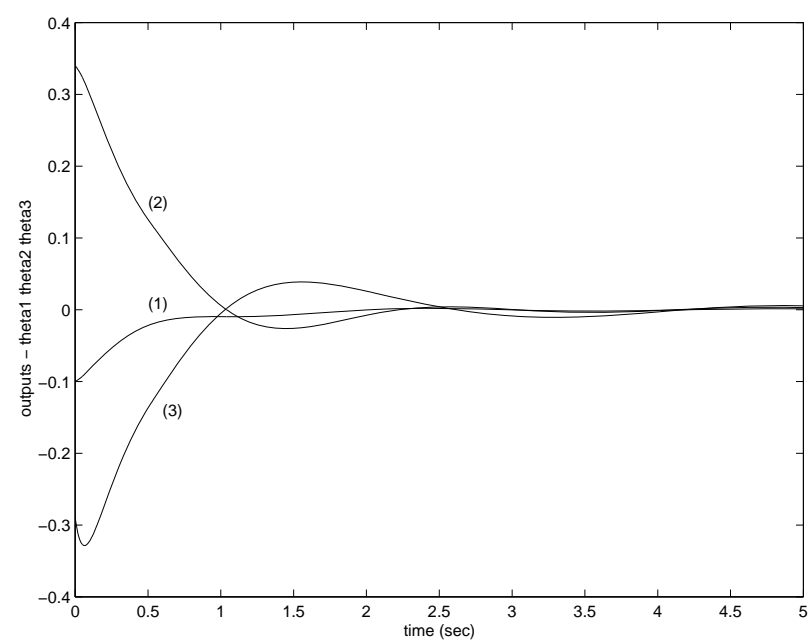

Figure 6 Outputs of Three Pendulums

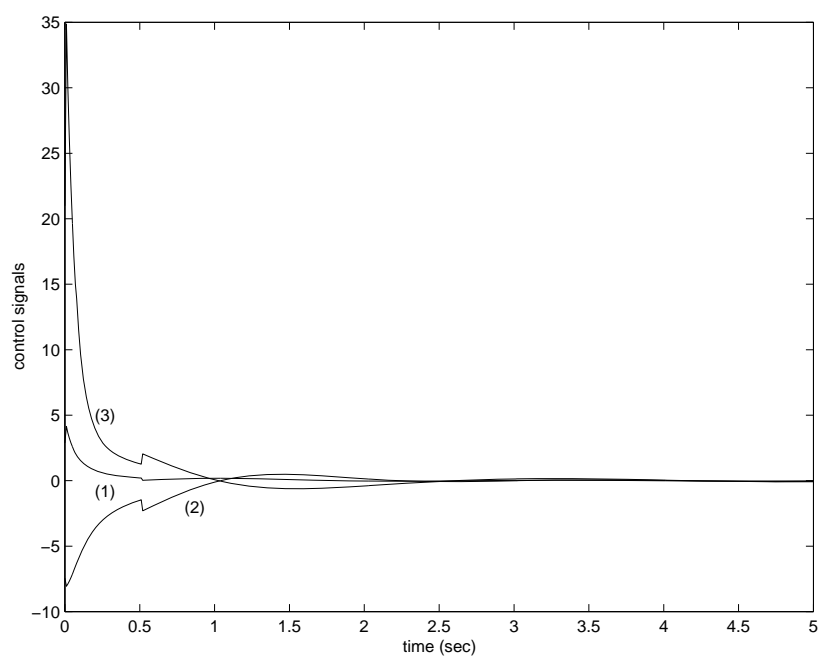

Figure 7 Control Signals

\section{Conclusions}

We ensure the stability of a MIMO system by establishing LMI conditions when the system is subject to degradation of actuators and sensors. The theory developed in Section 3 is demonstrated by three inverted coupled pendulums, which show the system can be stabilized.

\section{Acknowledgment}

This work was supported by the Center for Automation and Intelligent Research and DARPA/ARO grant.

\section{Appendix A}

\section{Proof.}

We consider the quadratic Lyapunov function $V(x)=$ $x^{T} L x$. The consideration of perturbation will be incorporated in the derivative of Lyapunov function for stabilization of overall system. We have

$$
\dot{\dot{V}}(x)+\sum_{r=1}^{m} \lambda_{r}\left(q_{u}^{r T} q_{u}^{r}-p_{u}^{r^{T}} p_{u}^{r}\right)<0
$$

for some $\lambda_{r}>0$. Substituting (2.1) into (A.1) and rearranging the expression, we obtain

$$
\left.\left[\begin{array}{c}
x \\
p_{u}
\end{array}\right]^{T}\left[\begin{array}{cc}
A^{T} L+L A+L B K+(L B K)^{T} \\
+K^{T} \varphi_{u}^{T} \Lambda \varphi_{u} K & L B \\
B^{T} L & -\Lambda
\end{array}\right] \begin{array}{l}
x \\
p_{u}
\end{array}\right]<0
$$

where the $\Lambda=\operatorname{diag}\left(\lambda_{1}, \lambda_{2}, \ldots, \lambda_{m}\right)$. (A.2) is equivalent to

$$
\left[\begin{array}{cc}
A^{T} L+L A+L B K+(L B K)^{T} \\
+K^{T} \varphi_{u}^{T} \Lambda \varphi_{u} K & L B \\
B^{T} L & -\Lambda
\end{array}\right]<0
$$

Expanding by Schur complement and letting $Q=L^{-1}$, $Y=K Q$, and $M=\Lambda^{-1}$, we may have

$$
\left[\begin{array}{cc}
Q A^{T}+A Q+B Y+Y^{T} B^{T}+B M B^{T} & Y^{T} \varphi_{u}^{T} \\
\varphi_{u} Y & -M
\end{array}\right]<0
$$

This completes the proof.

\section{Appendix B}

Proof.

We need the following lemma used in the proof.

Lemma B ([8]) For any matrices $M$ and $N$ with appropriate dimensions, we have

$$
M^{T} N+N^{T} M \leq M^{T} S N+N^{T} S^{-1} M
$$

for any positive-definite symmetric matrices $S$.

We consider the quadratic Lyapunov function $V(x)=$ $x^{T} L x$. The system perturbation will be incorporated in the derivative of Lyapunov function for stabilization of overall system. We have

$$
\dot{V}(x)+\sum_{r=1}^{n} \lambda_{r}\left(q_{s}^{r^{T}} q_{s}^{r}-p_{s}^{r^{T}} p_{s}^{r}\right)+\sum_{r=1}^{m} \xi_{r}\left(q_{u}^{r^{T}} q_{u}^{r}-p_{u}^{r^{T}} p_{u}^{r}\right)<0
$$


for some $\lambda_{r}>0$ and $\xi_{r}>0$. Substituting (3.6) into (B.1) and rearranging the expression, we obtain

$$
\left(\begin{array}{l}
x^{T}\left(A_{k}^{T} L+L A_{k}+K \varphi_{u}^{T} \Gamma \varphi_{u} K+\psi_{s}^{T} \Lambda \psi_{s}\right) x \\
+x^{T}\left(L B K+K^{T} \varphi_{u}^{T} \Gamma \varphi_{u} K\right) p_{s}+x^{T} L B p_{u} \\
+p_{s}^{T}\left(K^{T} B^{T} L+K^{T} \varphi_{u}^{T} \Gamma \varphi_{u} K\right) x+p_{u}^{T} B^{T} L x \\
+p_{s}^{T}\left(-\Lambda+K^{T} \varphi_{u}^{T} \Gamma \varphi_{u} K\right) p_{s}-p_{u}^{T} \Gamma p_{u}
\end{array}\right)<0
$$

where the $\Lambda=\operatorname{diag}\left(\lambda_{1}, \lambda_{2}, \ldots, \lambda_{m}\right)$ and $\Gamma=\operatorname{diag}\left(\xi_{1}, \xi_{2}, \ldots\right.$, $\left.\xi_{m}\right)$. Use of Lemma B we have

$$
\begin{gathered}
x^{T} L B p_{u}+p_{u}^{T} B^{T} L x \leq x^{T} L S_{u} L x+p_{u}^{T} B^{T} S_{u}^{-1} B p_{u} \\
x^{T} L B K p_{s}+p_{s}^{T} K^{T} B^{T} L x \leq x^{T} L B S_{x} B^{T} L x+p_{s}^{T} K^{T} S_{x}^{-1} K p_{s}
\end{gathered}
$$

$x^{T} K^{T} \Phi_{\Gamma}^{T} K p_{s}+p_{s}^{T} K^{T} \Phi_{\Gamma}^{T} K x \leq x^{T} K^{T} \Phi_{\Gamma}^{T} S_{s} \Phi_{\Gamma}^{T} K x+p_{s}^{T} K^{T} S_{s}^{-1} K p_{s}$ where $\Phi_{\Gamma}=\varphi_{u}^{T} \Gamma \varphi_{u}$. Thus,

(B. 2$) \leq$

$\left(\begin{array}{l}x^{T}\left(\begin{array}{l}A_{k}^{T} L+L A_{k}+K^{T}\left(\Phi_{\Gamma}+\Phi_{\Gamma}^{T} S_{s} \Phi_{\Gamma}\right) K+\psi_{s}^{T} \Lambda \psi_{s} \\ +L\left(S_{u}+B S_{x} B^{T}\right) L\end{array}\right) x \\ +p_{s}^{T}\left(-\Lambda+K^{T}\left(S_{x}^{-1}+S_{s}^{-1}+\Phi_{\Gamma}\right) K\right) p_{s}+p_{u}^{T}\left(-\Gamma+B^{T} S_{u}^{-1} B\right) p_{u}\end{array}\right)<0$

Therefore, we require

$$
\begin{gathered}
\left(\begin{array}{c}
A_{k}^{T} L+L A_{k}+K^{T}\left(\Phi_{\Gamma}+\Phi_{\Gamma}^{T} S_{s} \Phi_{\Gamma}\right) K \\
+\psi_{s}^{T} \Lambda \psi_{s}+L\left(S_{u}+B S_{x} B^{T}\right) L
\end{array}\right)<0 \\
-\Lambda+K^{T}\left(S_{x}^{-1}+S_{s}^{-1}+\Phi_{\Gamma}\right) K<0 \\
-\Gamma+B^{T} S_{u}^{-1} B<0
\end{gathered}
$$

Multiplying $P=L^{-1}$ on both side of (B.4) and applying Schur complement, we obtain

$$
\left[\begin{array}{ccc}
P A^{T}+A P+W^{T} B^{T}+B W & & W^{T} \\
+B S_{x} B^{T}+S_{u} & P \psi_{s}^{T} \\
W & -\left(\Phi_{\Gamma}^{T} S_{s} \Phi_{\Gamma}+\Phi_{\Gamma}\right)^{-1} & 0 \\
\psi_{s} P & 0 & -\Lambda^{-1}
\end{array}\right]<0
$$

where $W=K P$. Multiplying $P$ on both side of (B.5) we have

$$
-P \Lambda P+W^{T}\left(S_{x}^{-1}+S_{s}^{-1}+\Phi_{\Gamma}\right) W<0
$$

[1] R.J. Veillette, J.V. Medanic, and W.R. Perkins, "Design of Reliable Control Systems," IEEE Transactions on Automatic Control, vol. 37, No.3, pp.290-304, March 1992.

[2] M. Fujita and E. Shimenura, "Integrity Against Arbitrary Feedback-loop Failure in Linear Multivariable Control Systems," Automatica, vol. 24, No.6, pp. 765-772, 1988.

[3] M. Vidyasagar and N. Viswanadham, "Reliable Stabilization Using A Multi-controller Configuration," Automatica, vol.21, No.5, pp. 599-602, 1985.

[4] Y.J. Cho, Z. Bien, and B.K. Kim, "Reliable control via additive redundant adaptive control," in Proc. 1989 Amer. Contr. Conf., Pittsburgh, PA., pp. 1899-1904, 1989.

[5] C.C. Feng and S. Phillips, "Robust Decentralized Control; A Linear Matrix Inequality (LMI)-based design," Submitted to the 2017 CDC.

[6] S. Boyd and C.H. Barratt, Linear Controller Design - Limit of Performance. Prentice-Hall International, Inc. 1991.

[7] S. Boyd, L. El Ghaoui, E. Feron, and V. Balakrishnan, Linear Matrix Inequalities in System and Control Theory. SIAM books, Philadelphia, 1994.

[8] K. Zhou and P.P. Khargonekar, "Robust Stabilization of Linear Systems with Norm-Bounded Time-Varying Uncertainty," Systems and Control Letters, Vol. 10, pp. 17-20.

This completes the proof.

\section{References}

